\title{
Morphological changes in the corpus callosum: A study using joint Riemannian feature spaces
}

\author{
Meena Mani ${ }^{1, *}$, Anuj Srivastava ${ }^{2}$ and Christian Barillot ${ }^{1}$ \\ 1 VisAGeS Project, IRISA/INRIA F-35042, Rennes, France \\ ${ }^{2}$ Department of Statistics, Florida State University, Tallahassee, Florida, USA
}

\begin{abstract}
Shape, scale, orientation and position, the physical features associated with white matter DTI tracts, can either individually or in combination be used to define feature spaces. Recent work by Mani et al. ${ }^{1}$ describes a Riemannian framework in which these joint feature spaces are considered. In this paper, we use the tools and metrics defined within this mathematical framework to study morphological changes due to disease progression. We look at sections of the anterior corpus callosum, which describes a deep arc along the mid-sagittal plane, and show how multiple sclerosis and normal control populations have different joint shape-orientation signatures.
\end{abstract}

Keywords: Riemannian metrics, shape analysis, DTI, multiple sclerosis

\section{INTRODUCTION}

The corpus callosum (CC) connects the two brain hemispheres and thus plays a vital role in interhemispheric

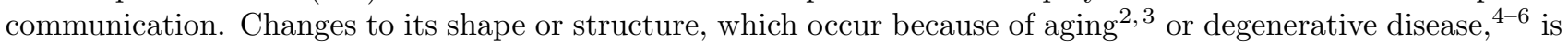
the subject of active study. There is an interest in linking these physical changes with neurological impairment. ${ }^{7}$ There is also an interest in linking the different stages of a degenerative disease such as multiple sclerosis (MS), schizophrenia or Alzheimer's, with physical alterations.8,

One of the underlying mechanisms for these topographical changes is atrophy. In the case of multiple sclerosis (MS), a degenerative disease associated with atrophy, whole brain grey matter volume loss occurs even in the earliest stages of the disease. What is not as well understood is the nature and extent of white matter volume loss. ${ }^{10}$ The CC is an ideal brain structure to study white matter atrophy in MS subjects since it has the largest concentration of white matter tracts and we expect it to be disproportionately affected.

The study of morphological changes in the CC, which falls under the purview of shape analysis, can be categorized as volume-based or tract-based. With volume-based analysis, two broad approaches have been followed in the literature. The first approach has been to present the $\mathrm{CC}$ as an abstraction using a geometric representation such as a fixed topology skeleton or a medial axis description. Classification using a linear discriminant method such as support vector machine (SVM) is then performed. ${ }^{11,12}$ In other studies, different principal component analysis (PCA) techniques have been used to study the modes of local and global variation. ${ }^{13}$ The second set of approaches, more common in clinical studies, has been to divide the CC into well-defined partitions and to analyze quantitive parameters such as the width (dorsal-to-ventral) and thickness (anterior-toposterior $)^{8,14,15}$ of these sections.

With tract-based analysis, the research emphasis has been on the evaluation of microstructure functions such as fractional anisotropy (FA) and mean diffusivity (MD) along the lengths of tracts. Correspondence and averaging methods have been applied to preserve the FA function for bundles and atlases of prominent $\mathrm{CC}$ tracts like the genu and splenium. ${ }^{16,17}$ Classification based on the geometrical shapes of fiber tracts has received less attention.

In this paper, we present new tract-based methods based on geometry to assess fiber integrity. We use the Riemannian metrics described in an earlier paper ${ }^{1}$ to look at changes in the shape and alignment of fiber tracts

* Present address: Center for Magnetic Resonance Research, University of Minnesota Medical School, Minneapolis, MN, U.S.A.

Correspondence: Meena Mani; e-mail: mmani@umn.edu 
and through a case-control study of MS subjects, demonstrate the feasibility of our methods. In particular, we look at the anterior $\mathrm{CC}$ which when viewed along the midsagittal plane forms a deep arc. We expect that atrophy would result in a straightening of this arc as some studies ${ }^{13}$ suggest. We also expect that the shape and orientation metrics we use, when considered together, are well suited to detect such changes.

Section 2 briefly describes these metrics. The results in the form of distance maps are presented in Section 3 and this is followed by a discussion in Section 4.

\section{MATHEMATICAL FRAMEWORK}

In previous work, ${ }^{1}$ we presented a Riemannian framework where the different physical features (shape, orientation, scale and position) associated with a fiber tract modeled as an open curve, could be studied. With this versatile setup, different combinations of features (shape, shape+orientation, shape+scale, shape+orientation+scale, shape+orientation+scale+position) are possible and each constitute a different joint manifold. Geodesic distances and geodesic paths computed between two curves quantify the differences between fibers. The main ideas in this framework are summarized below:

The fiber tract is represented as an open continuous parameterized curve, $\beta:[0,1] \rightarrow \mathbb{R}^{3}$. We define a representation of a curve $\beta$ using a square-root velocity function (SRVF): ${ }^{18}$

$$
q(t)=\frac{\dot{\beta}(t)}{\sqrt{\|\dot{\beta}(t)\|}}, \quad q:[0,1] \rightarrow \mathbb{R}^{3} .
$$

In order to compare any two curves, we will compare their SRVFs. The metric that we use for this comparison

is the $\mathbb{L}^{2}$ metric. One important advantage of this representation is its invariance to reparameterization of curves. Because of this invariance, we can define a distance between the two curves as

$$
d\left(\beta_{1}, \beta_{2}\right)=\min _{\gamma \in \Gamma}\left(\left\|q_{1}-\left(q_{2}, \gamma\right)\right\|_{2}\right),
$$

where $\gamma$ is the reparameterization function for any curve and $(q, \gamma)$ is the reparameterized SRVF. This minimization is performed using the standard dynamic programming (DP) algorithm, and it results in a quantification of differences in curves represented in the feature spaces.

In analyzing shape changes to the CC, we use metrics that quantify the shape and shape+orientation differences between curves.

\subsection{Comparison using shape and orientation}

Curves in the shape+orientation manifold are invariant to translation/position and scale. Since the SRVF function (Equation 1) is defined in its entirety by the derivative of $\beta$, translational invariance is automatically achieved. In order to remove the influence of scales of curves in this setup, we rescale them to be of the same length; a length of 1 is generally used. The set of all SRVF functions associated with curves of length one are elements of a unit hypersphere in $\mathbb{L}^{2}$ (since their norms are one). The differential geometry of a sphere is well-known so this simplifies the subsequent shape analysis; for example, if $q_{1}$ and $q_{2}$ are two elements of a unit hypersphere, the geodesic distance between them is given by length of shortest arc connecting them on the sphere. The distance between two curves that depends only on their shapes and orientations is:

$$
d_{1}\left(\beta_{1}, \beta_{2}\right)=\min _{\gamma \in \Gamma}\left(\cos ^{-1}\left(\int_{0}^{1}\left\langle\left(q_{1}, \gamma\right)(t),\left(q_{2}, \gamma\right)(t)\right\rangle d t\right)\right) .
$$

The quotient shape+orientation space is a sphere so the geodesic or shortest path between the two curves is a great circle. It can be specified analytically by

$$
\psi(\tau)=\frac{1}{\sin (\theta)}\left[\sin (\theta-\tau \theta) q_{1}+\sin (\tau \theta)\left(q_{2}, \gamma^{*}\right)\right]
$$

where $\theta=d_{1}\left(\beta_{1}, \beta_{2}\right)$. 


\subsection{Comparison using shape only}

To compare fibers based only on their shapes, we remove all other physical variables (positions, scales, and orientations) from the curve representation. We have already described how to achieve translation and scale invariance. In order to obtain invariance to orientation we define an equivalence class of functions under $\mathrm{SO}(3)$ (the set of all possible rotations in $\mathbb{R}^{3}$ ). Since we also have to be invariant to reparameterization, the group action of $\mathrm{SO}(3)$ is combined with the action of $\Gamma$ to obtain the shape space. Thus, we minimize over $S O(3)$ as well as $\Gamma$ to get the distance function

$$
d_{2}\left(\beta_{1}, \beta_{2}\right)=\min _{\gamma \in \Gamma, O \in S O(3)} \cos ^{-1}\left(\int_{0}^{1}\left\langle\left(q_{1}, \gamma\right)(t), O\left(q_{2}, \gamma\right)(t)\right\rangle d t\right) .
$$

Let $\gamma^{*}$ and $O^{*}$ be the reparameterization and the rotation that minimize the right side in this equation. For $\theta=d_{2}\left(\beta_{1}, \beta_{2}\right)$, the geodesic path between two curves is given by

$$
\psi(\tau)=\frac{1}{\sin (\theta)}\left[\sin (\theta-\tau \theta) q_{1}+\sin (\tau \theta)\left(O^{*} q_{2}, \gamma^{*}\right)\right] .
$$

\subsection{Computing means of fiber bundles}

To find a representative mean of a set of curves in a nonlinear manifold, we compute a Karcher mean. This is an intrinsic computation that uses the exponential and inverse exponential maps of the given manifold to compute a local mean. For a given collection of fibers $\left\{\beta_{1}, \beta_{2}, \ldots, \beta_{n}\right\}$, with function representations $\left\{q_{1}, q_{2}, \ldots, q_{n}\right\}$, the Karcher mean is defined as:

$$
\bar{\mu}_{n}=\underset{f \in \mathcal{S}}{\operatorname{argmin}} \sum_{i=1}^{n} d_{s}\left(q, q_{i}\right)^{2} .
$$

A gradient approach such as the gradient descent algorithm is used to find the local minimum of the cost function. The mean of a fiber bundle is a statistical summary, but also, as used in our data analysis, a convenient way to represent a set of fibers.

\section{RESULTS}

\subsection{Data}

To study shape changes in the genu, DTI data from 20 subjects, 10 normal controls (NC) and 10 MS patients were used. The MS subjects had a mean age of $44 \pm 12$ years and the mean disease duration was $10 \pm 10$ years. Registration of the data set is not required (other than an alignment to a common coordinate system) but was performed in this case. To obtain the DTI tracts, anatomical regions corresponding to the genu and rostrum were seeded and streamline tractography with fourth order Runge-Kutta integration was performed. A few fibers from the posterior-most section of the genu and another small set from the mid-section of the genu, were used to compute two average curves, one for each section. The Karcher mean algorithm (Equation 7) was used for this. We shall refer to these two curves, shown in Figure 1a, as the lower curve (LC) and the upper curve (UC) respectively. A total of $40 \mathrm{LC} / \mathrm{UC}$ curves $(2$ curves/subject $\times 10$ subjects/group $\times 2$ groups $)$ were used in this study. To maintain consistency, the entire dataset was post-processed by a single individual.

\subsection{Distance maps}

Distance matrices of the NC and MS populations are used to look at LC-LC, UC-UC and LC-UC distances. The layout of such a map is shown in Figure 1b. The first quadrant shows LC-LC distances of the 10 LC fibers in the group; the fourth quadrant shows the UC-UC distances of the $10 \mathrm{UC}$ fibers; the second and third quadrants show the LC-UC distances between 20 fibers. We represent both shape and shape+orientation distances and NC and MS populations in this way. The distances have a common scale so intra- and interpopulation comparisons can easily be made.

Distance maps are an efficient way to make intra- and interpopulation comparisons. We can analyze the three distribution profiles-the LC-LC, UC-UC and LC-UC distances-both within the NC and MS populations as well as between the NC-MS populations. We can also make the same comparisons between the two manifolds. 


\subsubsection{Shape distances}

From Figure 2 we see that the intrapopulation shape differences are small for the LC-LC/UC-UC comparisons. The LC-UC distances are only slightly greater. This pattern is observed in both the control and MS populations with the MS group having slightly larger differences. This suggests that the shapes of the two curves within a population are similar, with the MS population showing more variation. The variances in Table 1 computed for the shape manifold further support this visual summary.

\subsubsection{Shape+orientation distances}

The shape distances are a baseline. With the additive orientation distances in the shape+orientation manifold, we expect the LC-UC distances to be greater since the two curves are differently oriented. This is what we observe in the control group with large distances in the second and third quadrants (see Figure 2). The striking visual showing the second quadrant differences between the two manifolds is further supported by the Wilcoxon signed-rank statistic. This is a nonparametric hypothesis test between paired dependent samples. The $p$-value of 0 that we get for both the $\mathrm{NC}$ and MS populations strongly indicates that the median distances are different for the two manifolds.

Figure 2 also shows that the second quadrant LC-UC distances in the shape+orientation manifold are both smaller and more variable for MS patients. The LC-UC variance for the MS subjects in Table 1 in fact shows that they are the most variable group. Because of the heterogeneity in variance between the NC and MS groups, a nonparametric rank-based test to compare populations was not performed.

Overall, the data suggests that the relative LC and UC orientation differences become smaller for the MS group as the disease progresses.

\section{DISCUSSION/CONTRIBUTION}

In a comparative study of $\mathrm{NC}$ and MS subjects, the shape+orientation distance maps have different signatures for the two populations with the MS group showing more variability. This variability is suggestive of alterations to callosal shape that accompany illness progression. The genu curvature may either be getting more pronounced or straightening out. PCA models of shape changes in the CC indicate that the CC flattens out. ${ }^{13}$ We can confirm this by designing an experiment with three curves from three different locations in the anterior CC. More expensive longitudinal studies that follow a patient over time, can also verify this.

Using only $10 \mathrm{MS}$ subjects and $10 \mathrm{NC}$ for comparison, we have demonstrated an effective new design that uses shape distances in tandem with shape+orientation distances to study shape, morphology and changes to these. Our feasibility study needs to be repeated on a larger sample matched for age, gender and handedness, factors that are known to affect callosal size and shape. ${ }^{2,19}$ The broader context of this work is to develop methods to identify and track progressive white matter disease. We envision that tools such as this will improve the clinical evaluation of patients suffering from MS and other similar degenerative diseases.

Table 1. Within-group variance for distance distributions

\begin{tabular}{|c||c|c|c||c|c|c|}
\hline \multicolumn{1}{|c||}{} & \multicolumn{3}{c||}{$N C$} & \multicolumn{3}{c|}{$M S$} \\
\cline { 2 - 7 } & LC-LC & UC-UC & LC-UC & LC-LC & UC-UC & LC-UC \\
\hline \hline shape & 0.0026 & 0.0048 & 0.0030 & 0.0089 & 0.0039 & 0.0089 \\
shape+orientation & 0.0032 & 0.0043 & 0.0040 & 0.0080 & 0.0108 & 0.0176 \\
\hline
\end{tabular}

$\dagger$ Distances between the same curve $(\mathrm{d}=0)$ were not included in the variance computation

\section{REFERENCES}

[1] Mani, M., Kurtek, S., Barillot, C., and Srivastava, A., "A Comprehensive Riemannian Framework for the Analysis of White Matter Fiber Tracts," in [International Symposium on Biomedical Imaging (ISBI)], 1101-1104, IEEE, Rotterdam (April 2010). 


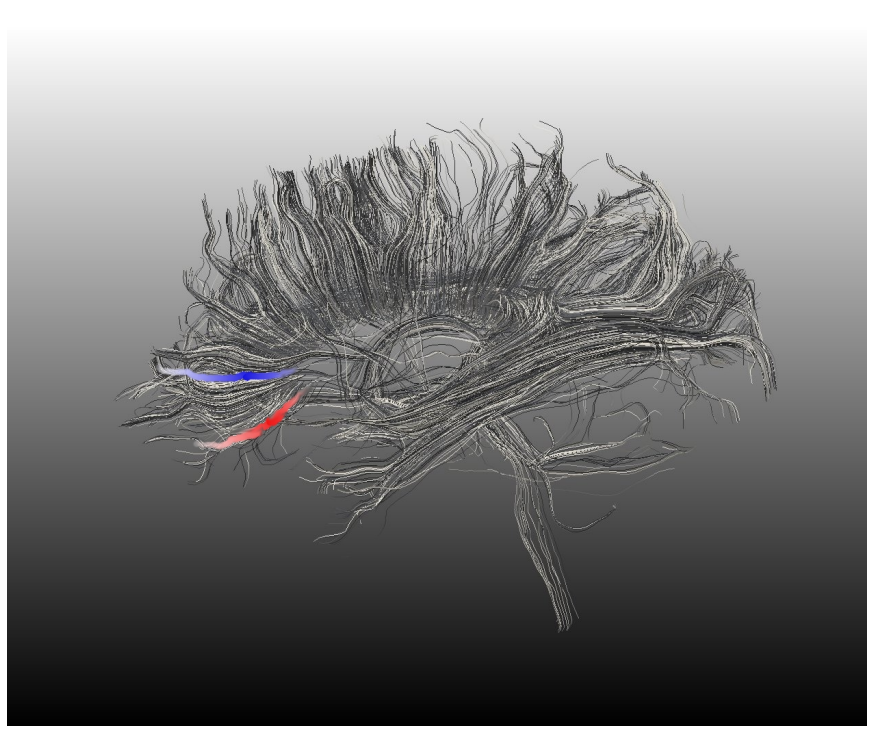

a

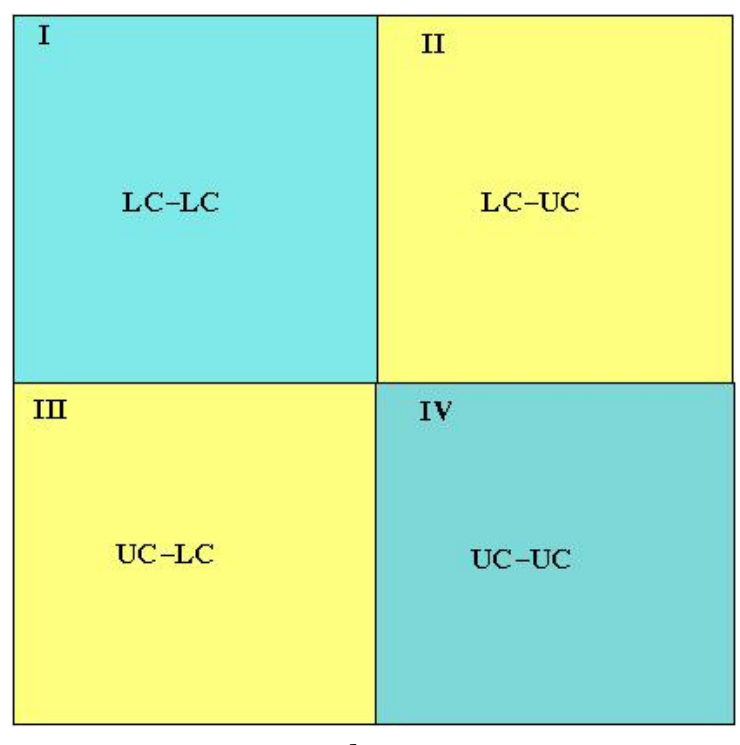

b

Figure 1. (a) The lower curve (LC) in red and the upper curve (UC) in blue are used to study curvature changes. (b) Quadrant I represents the pair-wise distances of the 10 LCs. Quadrant IV, displays the UC distances. Quadrants II/III show the LC-UC distances.

[2] Aboitiz, F., Rodriguez, E., Olivares, R., and Zaidel, E., "Age-related changes in fibre composition of the human corpus callosum: sex differences," NeuroReport 7(11), 1761-1764 (1996).

[3] Sullivan, E. V., Adalsteinsson, E., and Pfefferbaum, A., "Selective age-related degradation of anterior callosal fiber bundles quantified in vivo with fiber tracking," Cereb. Cortex 16(7), 1030-1039 (2006).

[4] Hardan, A. Y., Minshew, N. J., and Keshavan, M. S., "Corpus callosum size in autism," Neurology 55(7), 1033-1036 (2000).

[5] Woodruff, P. W., McManus, I. C., and David, A. S., "Meta-analysis of corpus callosum size in schizophrenia," Journal of Neurology, Neurosurgery and Psychiatry 58, 457-461 (1995).

[6] Simon, J. H., Jacobs, L. D., Campion, M. K., Rudick, R. A., Cookfair, D. L., Herndon, R. M., Richert, J. R., Salazar, A. M., Fischer, J. S., Goodkin, D. E., Simonian, N., Lajaunie, M., Miller, D. E., Wende, K., Martens-Davidson, A., Kinkel, R. P., Munschauer, F. E., I., and Brownscheidle, C. M., "A longitudinal study of brain atrophy in relapsing multiple sclerosis," Neurology 53(1), 139-148 (1999).

[7] Hampel, H., Teipel, S. J., Alexander, G. E., Horwitz, B., Teichberg, D., Schapiro, M. B., and Rapoport, S. I., "Corpus Callosum Atrophy Is a Possible Indicator of Region- and Cell Type-Specific Neuronal Degeneration in Alzheimer Disease: A Magnetic Resonance Imaging Analysis," Arch Neurol 55(2), 193-198 (1998).

[8] Walterfang, M., Wood, A. G., Reutens, D. C., Wood, S. J., Chen, J., Velakoulis, D., McGorry, P. D., and Pantelis, C., "Morphology of the corpus callosum at different stages of schizophrenia: cross-sectional study in first-episode and chronic illness," The British Journal of Psychiatry 192(6), 429-434 (2008).

[9] Teipel, S. J., Bayer, W., Alexander, G. E., Zebuhr, Y., Teichberg, D., Kulic, L., Schapiro, M. B., Moller, H.-J., Rapoport, S. I., and Hampel, H., "Progression of Corpus Callosum Atrophy in Alzheimer Disease," Arch Neurol 59(2), 243-248 (2002).

[10] Bermel, R. A. and Bakshi, R., "The measurement and clinical relevance of brain atrophy in multiple sclerosis," Lancet Neurol 5, 158-170 (2006).

[11] Yushkevich, P., Pizer, S. M., Joshi, S., and Marron, J. S., "Intuitive, localized analysis of shape variability," in [In International Conference on Information Processing in Medical Imaging], 402-408, Springer-Verlag (2001).

[12] Golland, P., Grimson, W. E. L., and Kikinis, R., "Statistical shape analysis using fixed topology skeletons: Corpus callosum study," in [IPMI], 382-387 (1999). 

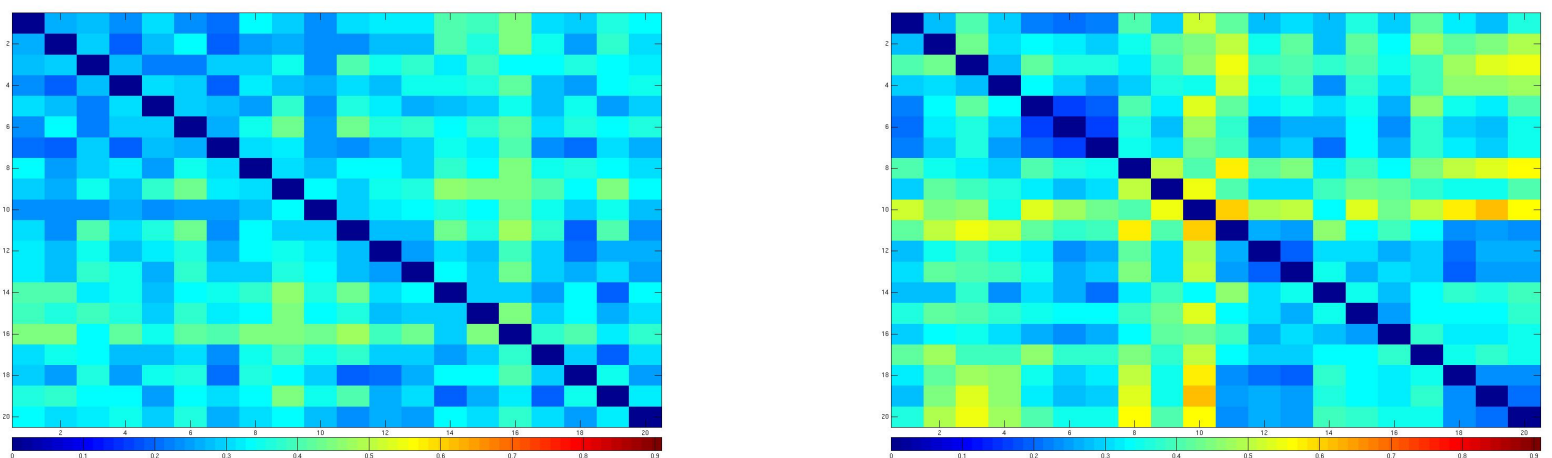

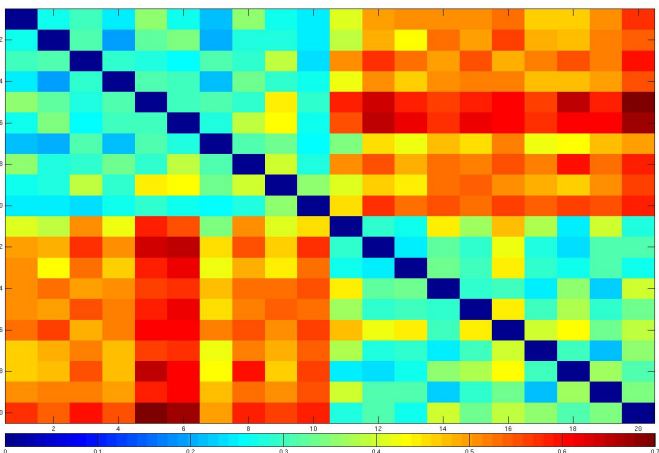

$\mathrm{NC}$

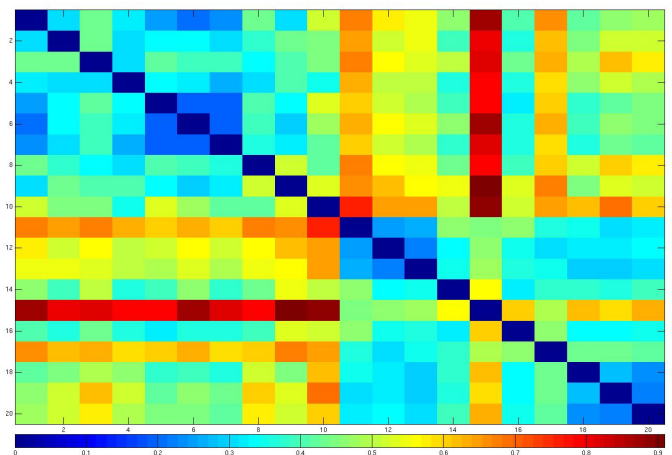

MS

Figure 2. Distance maps. Row 1: The LC-LC, UC-UC and LC-UC shape differences are small for the NC and MS populations. Row 2: The LC-UC shape+orientation distances are large (quadrants II \& III are orange) for the NC population. These distances are both smaller and more variable, by contrast, in the MS group. A common color scale enables us to compare groups.

[13] Ishaq, O., Hamarneh, G., Tam, R., and Traboulsee, A., "Longitudinal, regional and deformation-specific corpus callosum shape analysis for multiple sclerosis," in [International Conference of IEEE Engineering in Medicine and Biology Society (EMBC)], 2110-2113 (2007).

[14] Downhill, J. E., Buchsbaum, M. S., Wei, T., Spiegel-Cohen, J., Hazlett, E. A., Haznedar, M. M., Silverman, J., and Siever, L. J., "Shape and size of the corpus callosum in schizophrenia and schizotypal personality disorder," Schizophrenia Research 42(3), 193 - 208 (2000).

[15] Adluru, N., Hinrichs, C., Chung, M., Lee, J.-E., Singh, V., Bigler, E., Lange, N., Lainhart, J., and Alexander, A., "Classification in DTI using shapes of white matter tracts," in [International Conference of IEEE Engineering in Medicine and Biology Society (EMBC)], 2719 -2722 (2009).

[16] Corouge, I., Fletcher, P. T., Joshi, S., Gouttard, S., and Gerig, G., "Fiber tract-oriented statistics for quantitative diffusion tensor MRI analysis," in [Medical Image Analysis], 786-798 (2006).

[17] Goodlett, C. B., Fletcher, P. T., Gilmore, J. H., and Gerig, G., "Group analysis of DTI fiber tract statistics with application to neurodevelopment," NeuroImage 45(1, Supplement 1), S133 - S142 (2009). Mathematics in Brain Imaging.

[18] Joshi, S. H., Klassen, E., Srivastava, A., and Jermyn, I., "A novel representation for Riemannian analysis of elastic curves in $\mathrm{R}^{\mathrm{n}}$," in $[C V P R],(2007)$.

[19] Witelson, S. F., "Hand and Sex Differences in the Isthmus and Genu of the Human Corpus Callosum: A Postmortem Morphological Study," Brain 112(3), 799-835 (1989). 